\title{
Expansion and contraction of outline and solid acute angles: Effects of angle magnitude, type of display, and the nature of the matching task
}

\author{
PETER WENDEROTH, TONY O'CONNOR, and MICHAEL JOHNSON \\ University of Sydney, Sydney, New South Wales, Australia
}

\begin{abstract}
When a solid acute angle is reflected about its vertex to form a dual-angle display with a straight edge, the edge appears bent (the Bourdon illusion), in a manner which could be described as acute angle contraction. Smaller, but directionally similar, effects using outline angles have been reported by Rozvany and Day (1980). Since there is abundant evidence that single outline acute angles exhibit what can be described as acute-angle expansion, the following questions arise. First, do single solid angles induce angle contraction effects? Second, why do outline angles induce expansion effects in the single-angle display but not in the dual-angle display? Experiment 1 measured the angular function of tilt illusions induced by single outline and solid acute angles. In Experiment 2, which used dual-angle outline and solid displays, subjects set comparison lines parallel to either the upper or lower angle arm, or set a chevron to match the apparent bend in the whole edge formed by both arms. It was found that single angles show expansion when they are outline figures, contraction when they are solid, but that chevron settings in dual-angle displays cannot be predicted from parallel matches to the individual angle arms. These findings were consistent with the hypothesis that judgments in the domains of orientation, position, and collinearity reflect processing by parallel neural coding systems.
\end{abstract}

Converging evidence from a number of recent studies attests to the need to distinguish between various domains of perceptual judgment. This is so because certain measurements which are equivalent in Euclidean space are not equivalent, necessarily, in perceptual space. For example, although the individual bars of a grating must become thinner as its spatial frequency increases, observers nevertheless may judge an individual bar as thicker (Levinson \& Frome, 1979). Consequently, the perceptual domains of spatial frequency judgment and thickness judgment are not correlated in perceptual space in the same manner as they are in Euclidean space. The reason for this may be that the different tasks prompt the observer to attend to different aspects of the constant proximal stimulus; or an explanation may be sought in terms of separate neural mechanisms (or channels) concerned with processing frequency and thickness information.

Wenderoth (1983) showed that the identical stimulus display can result in either significant or nonsignificant errors of judgment, depending upon whether the observers' task is to position a given target stimulus in either the orientation or the position domain. Other experiments have demonstrated that observers' judgments in the orien-

This research was supported by a University of Sydney Special Projects grant in 1985, which funded the second author's Research Assistance. In 1986, support was provided by the Australian Research Grants Scheme, Grant A28515620 I, to the first author. We thank Geoff Stuart for drawing our attention to irradiation effects.

The authors' mailing address is: Department of Psychology, University of Sydney, Sydney, N.S.W., Australia. tation domain may not predict those in the angular domain, despite the fact that, in Euclidean terms, the physical size of an acute angle equals the difference in absolute orientation between the angle's arms (Emerson, Wenderoth, Curthoys, \& Edmonds, 1975; Wenderoth \& Johnson, 1984; Wenderoth \& White, 1979). Two recent studies have concluded that three perceptual domainsorientation, position, and collinearity (straightness)-are independent, at least in part. The evidence came from studies on the tilt illusion (Wenderoth \& Johnson, 1984) and the tilt aftereffect (Harris \& Calvert, in press). According to Harris and Calvert, the measured tilt aftereffect represents a neural compromise between separate neural channels coding orientation, position, and collinearity.

The purpose of the present study was to see whether this distinction among the domains of orientation, position, and straightness might help to explain some otherwise puzzling data on the Bourdon illusion (Figure 1).

When a straight line (AB in Figure 1a) is formed by reflecting a solid triangle (ACD) about its apex (C), the line does not appear straight. Rather, it is seen as a chevron bent in the same direction as the chevron formed by the other long sides of the triangles (DCE). This is the Bourdon effect, recently studied parametrically for the first time by Rozvany and Day (1980). In the course of their investigation, Rozvany and Day used additional stimulus displays, similar to the Bourdon figure, which were formed by outline triangles, with the short sides (AD, BE) either present (Figure 1b) or absent (Figure 1c). These outline figures induced smaller, but directionally similar, effects to the original, solid figure. 

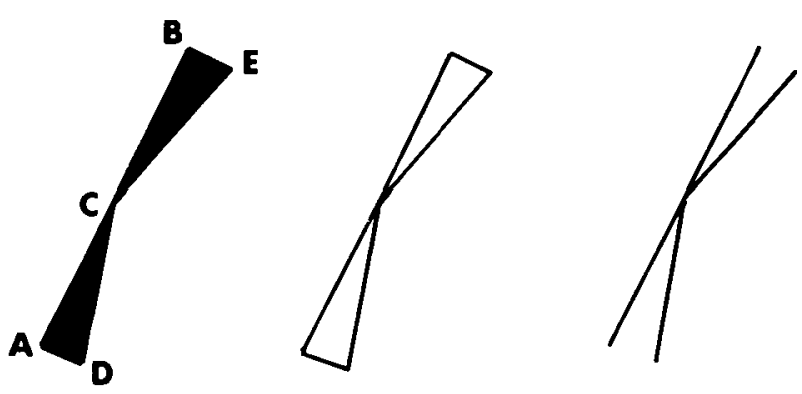

a

involving both the proposed collinearity (assimilation) effect and the separate tilt (contrast) effects. Hence, the collinearity or straightness judgments should show a small net effect which is not the simple sum of the orientation effects. These predictions were addressed by Experiment 2; Experiment 1 was concerned with a prior question.

In view of the above analysis, one reason that the solidangle Bourdon effect might be so much larger than the outline-angle effect could be that single solid angles do not exhibit contrast effects, or at least contrast effects as large as those induced by outline angles. In that event, the collinearity assimilation effect would dominate the judgment of the solid-angle figure, resulting in a larger illusion. Since no one has measured single-angle tilt illusions with solid angles, this was the aim of Experiment 1.

Figure 1. (a) The Bourdon illusion: Edge ACB is straight but appears to be a chevron bent in the same direction as DCE. (b, c) Variants of the Bourdon illusion studied by Rozvany and Day (1980).

This result seems surprising because single outline acute angles with subtense similar to those used by Rozvany and Day $\left(12.5^{\circ}\right)$ exhibit tilt contrast: the angles' arms repel each other in the orientation domain (e.g., Carpenter \& Blakemore, 1973; Gibson, 1937; O'Toole \& Wenderoth, 1977; Virsu \& Taskinen, 1975; Wenderoth \& Johnson, 1984). It might have been expected, therefore, that the straight edge, AB in Figure 1c, would appear bent in the direction opposite to the chevron DCE formed by the other lines. The questions, then, are: Why do single outline acute angles appear to produce contrast effects, whereas dual-angle displays appear to result in an assimilation effect? And why does the solid angle display (Figure 1a) induce an assimilation effect that is so much larger than that induced by the outline display (Figure 1c), with average illusions of approximately $2.6^{\circ}$ and $0.7^{\circ}$, respectively, in the Rozvany and Day experiments?

Let us assume, with Harris and Calvert (in press), that any stimulus is processed by three parallel systems that code orientation, position, and straightness. There is evidence that the apparent position of a stimulus may shift toward adjacent contours or objects (Hartley, 1978, 1979; Wenderoth, 1980). Assume, then, that the chevron in Figure $1 \mathrm{c}$ induces an assimilation in the straight test edge so that it appears bent in the same direction. Simultaneously, the individual acute-angle arms are coded as repelling each other in the orientation domain, a directionally opposite effect. The compromise between the orientation and collinearity information would result in a small net effect, as Rozvany and Day found. One approach to testing this hypothesis would be to have subjects judge the orientations of the individual angle arms in Figure 1c and also to judge the shape (collinearity) of the entire straight edge. If the above speculations are correct, then the orientation judgments should reveal contrast effects because the observer would be attending to only one arm of one of the angles in the display and judging orientation. In the case of the collinearity judgments, the observer would be required to compare the two individual angle arms,

\section{EXPERIMENT 1}

Given the knowledge that single outline acute angles exhibit tilt contrast and that the dual solid-angle Bourdon display shows what appears to be an assimilation effect, the question arises as to whether the major determinant of the Bourdon effect is the double angle or the solid nature of the individual angles. Although we knew of no experiments on the judged orientation of the arms of solid angles, some data were available on the effects of adding extra lines to the interiors of acute angles. For example, Carpenter and Blakemore (1973) reported that the addition of a third line within an acute angle reduced tilt contrast effects due, they supposed, to disinhibition. Rentschler, Hilz, Sutterlin, and Noguchi (1981) added fans of many lines within various acute angles and found that, regardless of overall angle size, maximum repulsion of the outermost arms occurred when lines in the fan were $15^{\circ}$ apart, the very angle which usually gives the largest tilt contrast effect. Adding more lines reduced the illusion but did not reverse it.

However, these studies may not bear on completely solid angle effects, because even the largest fans of lines used by Rentschler et al. had angles of $5^{\circ}$ between lines so that the individual lines were resolvable.

The purpose of Experiment 1, then, was to measure the judged orientation of solid-acute-angle arms for a range of angle sizes between $0^{\circ}$ and $90^{\circ}$, the so-called angular function of the solid-angle effect. It is well documented that the angular function of the outline acute-angle effect can be measured by having observers set a short comparison line ( $\mathrm{C}$ in Figure 2a) parallel to the test arm (T) of an acute angle, as the angle $(\theta)$ between $T$ and the other, inducing arm (I) varies. Because constant errors in parallel matching occur even in the absence of line $I$, it is necessary to include this $0^{\circ}$ condition as a pretest judgment, the mean of which is then subtracted from all test judgments for angles $>0^{\circ}$ (Carpenter \& Blakemore, 1973).

In Experiment 1, then, we used identical methods of parallel matching to measure the angular function of out- 

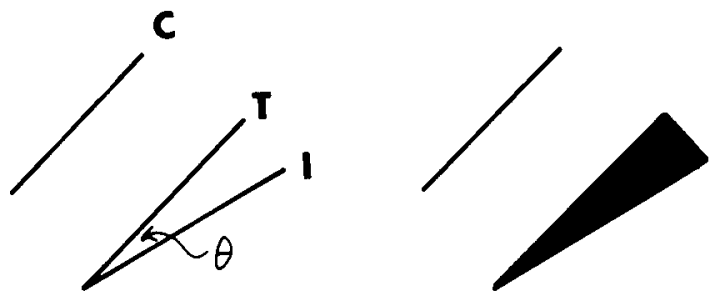

a

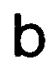

Figure 2. (a) The tilt illusion and its measurement: Inducing line (I) causes test line (T) to appear tilted more counterclockwise than it really is. The effect varies with angle $\theta$ and is measured by setting the comparison line (C) parallel to $T$ in the absence (pretest control) and in the presence of $I$. (b) Display for measuring tilt illusions induced by solid angles.

line acute angles (Figure 2a) and solid acute angles (Figure 2b), for angles ranging from $0^{\circ}$ to $90^{\circ}$ in $10^{\circ}$ steps.

\section{Method}

Apparatus. Stimuli were displayed on the flat screen of a Tektronix 608 monitor (P4 phosphor) which was interfaced with a Hewlett-Packard graphics translator (1350A) and a PDP-11/20 computer.

The outer two of three microswitches could be used by the subject to rotate a comparison line clockwise or counterclockwise in $0.25^{\circ}$ steps, to match the apparent orientation of an angle arm. A third, center switch indicated completion of adjustment, removed the display, and stored the result. The next stimulus was presented either immediately (outline angles) or after a variable delay (solid angles), depending upon how much time the computer required to draw the solid angle (average 10-20 sec).

Subjects viewed the display screen in a dark, windowless laboratory from a padded forehead- and chinrest $57 \mathrm{~cm}$ from the screen, so that $1 \mathrm{~cm}$ subtended $1^{\circ}$ of visual angle.

Stimuli. The stimulus angles were either outline or solid angles, as in Figures $2 \mathrm{a}$ and $2 \mathrm{~b}$. Solid angles were produced by 50 crosshatch vectors, each $0.1 \mathrm{~mm}$ thick, separated by $0.1 \mathrm{~mm}$. The $\mathrm{T}$ line always was oriented $45^{\circ}\left(0^{\circ}\right.$ : horizontal) and both $\mathrm{I}$ and $\mathrm{T}$ lines were $1^{\circ}$ in length. The angle between $I$ and $T$ varied from $0^{\circ}$ (I absent) to $90^{\circ}$, in $10^{\circ}$ steps. The $C$ line, which was $0.8^{\circ}$ long with its center $1.2^{\circ}$ orthogonally above the center of $T$, rotated about its center in $0.25^{\circ}$ steps, at an apparent rate of rotation of about $2 \% \mathrm{sec}$. Stimuli were viewed through a black circular outline mask and partially crossed Polaroid filters so that the luminance of the lines was about $1.8 \mathrm{~cd} / \mathrm{m}^{2}$ as measured by an SE1 photometer. Contrast, defined as $\left(\mathrm{L}_{\max }-\mathrm{L}_{\min }\right) /\left(\mathrm{L}_{\max }+\mathrm{L}_{\min }\right)$, was close to 1.0.

Procedure. Each subject received all conditions in a different random order: 10 angles $\times$ solid versus outline $\times$ four starting positions (SPs), $\pm 5^{\circ}$ and $\pm 10^{\circ}$ from true parallelism. It took about an hour, with voluntary rests, to complete all 80 judgments. Bracketing prior to making a final setting was encouraged.

Subjects. Nine subjects were volunteers from an introductory psychology course; the 10th was the first author. All had emmetropic or corrected vision.

\section{Results}

The angular functions obtained with outline and solid angles are shown in Figure 3, where each illusion is the difference between the mean test setting (complete angle present) and the pretest control condition ( $0^{\circ}$ angle) in which only the test line was present. The two circular symbols at $0^{\circ}$ in Figure 3 show the actual pretest means. Average standard errors were identical for outline- and solidangle effects (0.36); a \pm 1 standard error bar is shown for the $90^{\circ}$ solid-angle mean in Figure 3.

Clearly, the outline angles exhibited the standard angular function, with repulsion effects (i.e., + ve or CCW setting of line $\mathrm{C}$ ) peaking at $20^{\circ}$; the solid angles all induced attraction ( - ve) effects, with a less obvious peak also at $20^{\circ}$ and additional large negative effects at $40^{\circ}$ and $90^{\circ}$. A simple subjects $\times$ treatments analysis (Winer, 1962, chap. 4), with planned contrasts, was used to compare outline- and solid-angle illusions for each angle size. Solid and outline illusions differed significantly at $10^{\circ}(F=6.86$, $p<.01)$ and at $20^{\circ}(F=33.7, p<.0005), 30^{\circ}(F=$ $12.68, p<.0005), 40^{\circ}(F=13.12, p<.0005), 50^{\circ}(F$ $=6.94, p<.01)$, and $90^{\circ}(F=9.77, p<.005)$. (Degrees of freedom $=1,171$ in each case.) Differences at $0^{\circ}, 60^{\circ}, 70^{\circ}$, and $80^{\circ}$ were not significant $(F=0.18$, 3.86 , and 2.28 , respectively).

From Figure 3, it can be concluded that single outline acute angles exhibit contrast effects, with a peak in the angular function around $20^{\circ}$ when the T line is oriented $45^{\circ}$ (a well-established finding), whereas solid angles induce assimilation effects.

We hesitate to claim any more regarding the form of the solid-angle angular function for several reasons. First, the data in Figure 3 show the mean differences between test and pretest settings, but there is some debate in the literature as to whether the appropriate pretest control measure is that for a $0^{\circ}$ angle or that for a $90^{\circ}$ angle (Wenderoth \& Johnson, 1985). The choice makes little difference in the case of outline angles, where the two uncorrected means are at least directionally similar $\left(+0.42^{\circ}\right.$ and $+0.89^{\circ}$, respectively).

For the solid angles, however, the $0^{\circ}$ mean was $+0.20^{\circ}$ (and the stimulus was identical to the outline $0^{\circ}$ stimulus) whereas the $90^{\circ}$ mean was $-0.91^{\circ}$. Although the uncorrected $90^{\circ}$ means were similar in magnitude but directionally opposite for the outline and solid angles $\left(+0.89^{\circ}\right.$ and $-0.91^{\circ}$, respectively), the choice of which mean $\left(0^{\circ}\right.$ or $90^{\circ}$ ) to use as pretest was difficult. If the $0^{\circ}$ means are

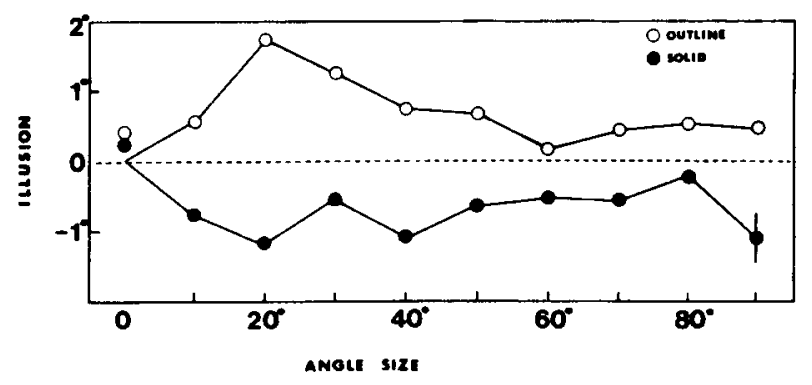

Figure 3. Angular functions of tilt illusions induced by outline angles (open symbols) and solid angles (filled symbols) in Experiment 1. Bar at $90^{\circ}$ shows \pm 1 standard error. Symbols at $0^{\circ}$ show obtained pretest means. Positive illusions mean repulsion of angle arms; negative illusions indicate attraction of arms. 
used as pretests, because they are positive, subtracting them results in a large and significant negative $90^{\circ}$ effect for solid angles; yet subtraction of the $90^{\circ}$ means as pretests would produce near zero effects for all solid angles.

It seemed possible that the negative $90^{\circ}$ solid-angle effect was due to the nature of the stimulus display. We were forced to produce solid angles by cross-hatching when other methods (e.g., fans of vectors originating at the vertex) produced undue distortion of angle arms. The cross-hatching method makes the solid angle a triangle. This is not a serious problem when the angle $\theta$ (Figure 2) is small, because the side opposite $\theta$ is very short and the difference between a straight edge and an arc is minimal. For large angles, such as $80^{\circ}$ or $90^{\circ}$, however, the T line is bounded by two angles, $80^{\circ}$ or $90^{\circ}$ at one end and $50^{\circ}$ or $45^{\circ}$, respectively, at the other. For these reasons, we wish to conclude only that small solid angles $\left(0^{\circ}-20^{\circ}\right)$ induce assimilation effects, and to conduct further investigation on larger solid angles, when angles can be produced as segments of a circle rather than triangles. Given these considerations, we chose to use the standard procedure of adopting the $0^{\circ}$ mean as pretest.

Finally, Pierce (1898) showed that a white right angle on a black ground appears less than a right angle, due to irradiation, the encroachment of light areas into dark areas, which is greater at corners than elsewhere. This effect could have contributed to the negative $90^{\circ}$ mean in Figure 3, but unfortunately we could not reverse contrast on the display to test the idea. However, in our displays there were corners (angles) at both ends of the test lines, so that a simple version of the irradiation hypothesis is unlikely to explain the results.

\section{Discussion}

The results of Experiment 1 were consistent with the hypothesis outlined in the introduction, namely that the solid-angle Bourdon effect shows larger assimilation effects than the outline display because individual solid angles induce less tilt contrast. Indeed, Experiment 1 suggests that individual solid angles induce tilt assimilation.

Experiment 2 was designed to obtain judgments from the same observers both of the apparent orientation of individual angle arms in outline and solid Bourdon displays and of apparent noncollinearity of the pairs of angle arms.

\section{EXPERIMENT 2}

In Experiment 2, observers, presented with outline or solid Bourdon displays, performed three tasks with each display. They were instructed to set an upper comparison line parallel to the upper angle's arm; to repeat this with a lower comparison line for the lower angle's arm; and then, with both comparison lines forming an adjustable chevron, to match the angle of the adjacent Bourdon edges. The three tasks are shown in Figure 4. (This matching method using a chevron [Figure 4] differs from the method used by Rozvany and Day [1980], who had subjects adjust the angle figure itself until the chevron a

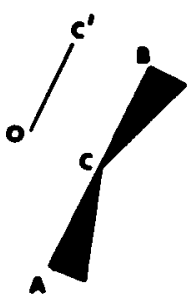

b

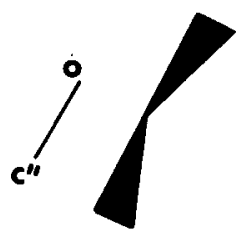

C

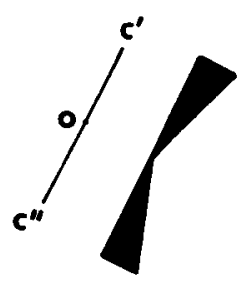

Figure 4. The three tasks given observers in Experiment 2. (a) Set upper comparison line $\left(\mathrm{OC}^{\prime}\right)$ parallel to edge $\mathrm{CB}$. (b) Set lower comparison line (OC") parallel to $C A$. (c) Set angle of chevron $\left(C^{\prime} O C^{\prime \prime}\right)$ to match angle of chevron BCA.

formed by the adjacent angle arms appeared straight. For solid angles, this required lines to be added so that the magnitude of the internal angles [BCE and ACD in Figure 1] varied with the subjects' settings, a confounding which seemed best avoided here.) There were 12 conditions in all: two angle sizes $\left(10^{\circ}\right.$ and $\left.20^{\circ}\right) \times$ three matching tasks $\times$ outline or solid angles.

\section{Method}

Apparatus. The apparatus was the same as that in Experiment 1. Stimuli. The stimuli were produced as in Experiment 1. The straight edge of the Bourdon figures (ACB in Figure 4) was oriented $67.5^{\circ}$, where Rozvany and Day (1980) obtained largest effects. All angle arms (e.g., AC, $\mathrm{CB}$ ) were $1^{\circ}$ long. Each comparison line $\left(O C^{\prime}\right.$ or $O C^{\prime \prime}$ in Figure 4) was $0.8^{\circ}$ long. Point 0 , the pivot of the comparison lines, was orthogonally directly aligned with point $\mathrm{C}$. The virtual perpendicular from $C^{\prime}$ (or $C^{\prime \prime}$ ) to $A B$ intersected it at $0.2^{\circ}$ short of $\mathrm{B}$ (or A). The chevron, $\mathrm{C}^{\prime} \mathrm{OC}^{\prime \prime}$ in Figure $4 \mathrm{c}$, was simply the sum of the upper and lower comparison lines in Figures $4 a$ and $4 b$.

There were three additional pretest (control) stimuli, consisting of a single straight line (ACB in Figure 4) and an upper or a lower comparison line, or both (the chevron). These control stimuli were judged by observers who were different from those who judged the angle figures (see Procedure).

Subjects. Thirty-one volunteers from an introductory psychology course were tested. Of these, 21 judged the 12 angle figures and 10 completed the pretest (control) measures. All subjects had emmetropic or corrected vision.

Procedure. Large and systematic constant errors occur in parallel matching a pair of lines, especially when the lines are oriented around $22.5^{\circ}$ from a main axis of space, as in this experiment (see Carpenter \& Blakemore, 1973). Such errors also vary with the relative positioning and lengths of the lines (see, e.g., Wenderoth \& Johnson, 1984). This meant that baseline (control) measures were needed in case errors in matching $O C^{\prime}, O C^{\prime \prime}$, and $C^{\prime} O C^{\prime \prime}$ differed significantly even when the test figure was simply a straight edge (ACB). However, because edge ACB was always physically straight, even in the angle displays, it was considered best not to include obviously straight pretest edges in the experimental trials. Partly for that reason, the control readings were taken from a separate group of 10 subjects.

For both experimental and control conditions, each stimulus display was judged four times, with comparison line starting positions of $\pm 5^{\circ}$ and $\pm 10^{\circ}$ from true parallelism. Thus, the 21 experimental subjects made 48 judgments in random order and the control subjects made 12 judgments. A second reason for using separate groups was that, due to the difficulty of the tasks, several experimen- 
tal subjects were only just able to complete the 48 trials in the $1 \mathrm{~h}$ provided.

\section{Results}

The mean pretest (control) settings of the 12 subjects given this task were $-1.19^{\circ}$ (standard error: 0.36), $+1.75(0.29)$, and $+0.21(0.44)$, respectively, for the three conditions shown in Figures $4 a, 4 b$, and $4 c$. The difference in algebraic sign between the first two conditions is misleading and arises from sign conventions adopted to measure illusions for the following reasons. First, whenever chevron illusions are reported, the error given is the deviation of one (the upper) chevron arm from collinearity, so that the total angular deviation is twice the reported error. Second, for the measurement of illusions, algebraic sign conventions were adopted such that repulsion (contrast) effects would be positive. In the case of the dual-angle Bourdon display, this required that a counterclockwise error be positive for a parallel match to the upper angle; that a clockwise error be positive for a match to the lower angle; and that a counterclockwise error be positive for the upper arm of the chevron. Consequently, the apparent directional difference between the parallel matching pretests means that the errors were in the same absolute direction: in both cases, the comparison line was set so that it and the test line converged at their upper ends, as found before with long test lines (Carpenter \& Blakemore, 1973). To test for differences between pretests, then, the algebraic sign of the upper match was reversed. Following a simple subjects $x$ treatments analysis as in Experiment 1, two planned contrasts showed that the mean parallel match to the upper portion of the line did not differ from that to the lower portion $[F(1,18)=1.09, p>.05]$. The mean of these two matches did differ from the mean error obtained with the chevron $(F=7.46, p<.025)$.

The appropriate pretest means were subtracted from each of the 21 experimental subjects' settings, and these corrected mean illusions are shown in Figure 5.

Parallel matches to the arms of the angles were as in Experiment 1: positive for outline angles (open circles and triangles) and negative for filled angles (solid circles and triangles). Chevron illusions were close to zero for outline angles (open squares) but large and negative for filled angles (solid squares).

Eleven planned contrasts were carried out, with the critical value of $F(1,220)=3.84$, for $p=.05$. Four contrasts compared upper and lower parallel matches. The difference between the upper and lower matches for the outline $10^{\circ}$ angles was significant $(F=3.91, p<.05)$, but the difference was not significant for the $20^{\circ}$ outline angles $(F=1.99)$ or for either of the corresponding solid angles ( $F=0.04$ and $F=0.61$, respectively).

Four more contrasts compared the average of the upper and lower parallel matches with the corresponding chevron matches. Three of these differences were significant: outline $10^{\circ}$ angles $(F=13.805, p<.0005)$, solid $10^{\circ}$ angles $(F=9.412, p<.005)$, and solid $20^{\circ}$ angles

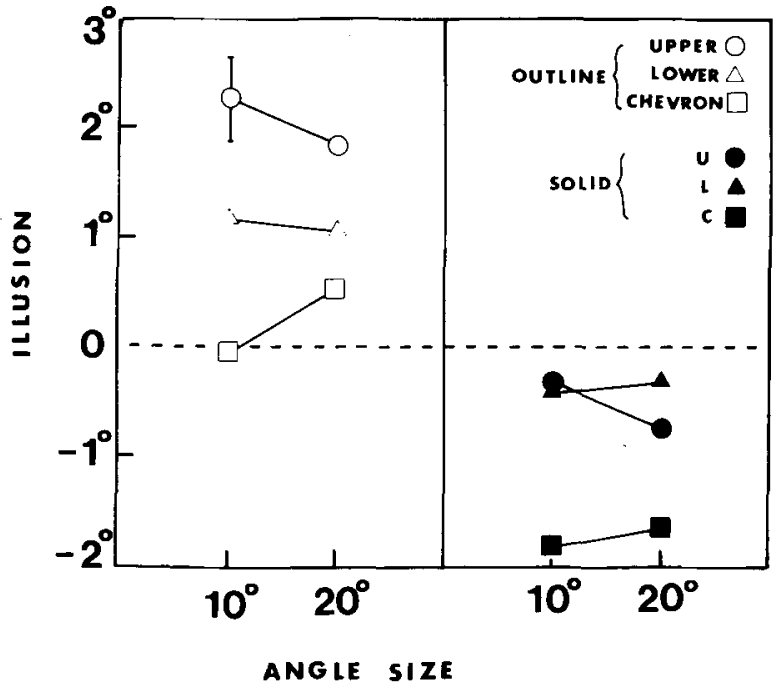

Figure 5. Mean tilt illusions, Experiment 2. Open symbols in left panel show outline-angle effects; filled symbols in right panel show solid angle effects. Parallel matches to upper angle arms are shown by circles, and those to lower angle arms by triangles. Chevron matches are shown by squares. Bar in upper left panel shows \pm 1 standard error. Positive illusions indicate repulsion effects.

$(F=6.031, p<.025)$. The difference for the outline $20^{\circ}$ angles was not significant $(F=3.77, p>.05)$.

The overall mean illusion for outline angles differed from that for solid angles, both when the angles were $10^{\circ}$ $(F=39.69, p<.0005)$ and when they were $20^{\circ}(F=$ $41.18, p<.0005)$. Finally, there was no significant difference between $10^{\circ}$ and $20^{\circ}$ illusions, averaged over angle type $(F=0.024, p>.05)$.

\section{GENERAL DISCUSSION}

The results of Experiments 1 and 2 generally were consistent with the findings of Rozvany and Day (1980), who reported that solid-angle Bourdon figures induced negative illusions of direction and that outline Bourdon figures induced near zero (slightly negative) illusions. The additional information from the present experiments is that single solid angles induce negative illusions when parallel matching is used, that such solid-angle illusions remain negative in the dual-angle display, and that outline-angle parallel matches also remain positive in the dual-angle display, but that chevron matches are not predictable from either the average of the upper and lower parallel matches ( 3 out of 4 significant contrasts) or their sum (inspection of data).

The results of these experiments are consistent with the hypothesis outlined earlier, namely that perceptual judgments reflect a compromise between separate neural channels coding orientation, position, and collinearity. Under normal conditions, relatively simple stimuli, such as vertical lines or gratings, are perceived veridically and no compromise is necessary between the signals generated in these three channels. Only under special circumstances, 
such as those presented by the outline-angle Bourdon display, do the signals from the three channels fail to coincide, requiring an output reflecting compromise.

It might be suggested that the near-zero effects obtained with chevron matching using the outline-angle display (open squares in Figure 5) reflect a cancellation by equal (or near equal) but opposite tilt illusions. That is, since the two right-hand angle arms in Figure $1 \mathrm{C}$ are oriented symmetrically around the collinear arms, they induce equal, but opposite, tilt illusions on those arms. However, this idea would predict no significant illusion for any of the three outline conditions in Figure 5 and therefore is untenable: only the chevron effect was near zero.

A final point relating to the outline Bourdon display is that some subjects asked, after the experiment, whether this display in fact did have a straight edge. It is possible that straightness or collinearity cues are more salient in the outline display. In a similar vein, Wenderoth and Johnson (1984) suggested that the arms of an acute angle may appear straight, even though parallel matching with a short comparison line reveals different perceived orientations of segments along the angle's arm. In support of this notion, Harris and Calvert (in press) proposed that orientation and position are coded by small, local mechanisms, whereas collinearity is a long-range (global) process.

Experiments are planned in which the two angles in solid and outline Bourdon displays are systematically separated in space. If there is a particular saliency of the straight edge in the outline figure, then separating the two angles might change chevron settings much more for the outline-angle than for the solid-angle figure. Other experiments will examine chevron matches to Bourdon figures in which the lower angle is not only reflected but also rotated, so that the test chevron is no longer physically straight.

Additional research is required on solid-angle tilt illusions to establish the precise angular function, to examine the effect (if any) of contrast reversal, and generally to establish how, if at all, solid-angle illusions are related to outline-angle illusions. Given that the outline tilt illusion is most frequently attributed to lateral inhibitory interactions between populations of orientation-selective neurons in cortical area V1, it would be of considerable interest to examine the responses of such cells to outline and solid angles positioned variously within their receptive fields.

\section{REFERENCES}

Carpenter, R. H. S., \& BlaKemore, C. (1973). Interactions between orientations in human vision. Experimental Brain Research, 18, 287-303.

Emerson, P., Wenderoth, P., Curthoys, I., \& Edmonds, I. (1975). Measuring perceived orientation. Vision Research, 15, 1031-1033.

Gibson, J. J. (1937). Adaptation, aftereffect and contrast in the perception of tilted lines: II. Simultaneous contrast and the areal restriction of the aftereffect. Journal of Experimental Psychology, 20, 553-569.

HARRIS, J. P., \& CALVERT, J. E. (in press). The tilt after-effect: Changes with stimulus size and eccentricity. Spatial Vision.

HaRTLEY, A. A. (1978). The major-axis effect: Axes of bilateral symmetry or loci of neural interactions? Perception \& Psychophysics, 23, 537-541.

Hartley, A. A. (1979). Major axes of stimulus figures do not account for dot displacements. Perception \& Psychophysics, 26, 331-332.

Levinson, J. Z., \& Frome, F. S. (1979). Perception of size of one object among many. Science, 206, 1425-1426.

OTOOLE, B., \& WeNderoth, P. (1977). The tilt illusion: Repulsion and attraction effects in the oblique meridian. Vision Research, 17, 367-374.

Pierce, A. H. (1898). The illusion of the kindergarten patterns. Psychological Review, 5, 233-253.

Rentschler, I., Hilz, R., Sutterlin, C., \& Noguchi, K. (1981). Illusions of filled lateral and angular extent. Experimental Brain Research, 44, 154-158.

Rozvany, G. E., \& Day, R. H. (1980). Determinants of the Bourdon effect. Perception \& Psychophysics, 28, 39-44.

VIRSU, V., \& TASKINEN, H. (1975). Central inhibitory interactions in human vision. Experimental Brain Research, 23, 65-74.

Wenderoth, P. (1980). Dot displacements can be deceptive: A reply to Hartley. Perception \& Psychophysics, 27, 368-369.

WENDEROTH, P. (1983). Identical stimuli are judged differently in the orientation and position domains. Perception \& Psychophysics, 33, 399-402.

WENDEROTH, P., \& Johnson, M. (1984). The effects of angle-arm length on judgments of angle magnitude and orientation contrast. Perception \& Psychophysics, 36, 538-544.

WENDEROTH, P., \& JoHNSON, M. (1985). What is the appropriate control for the tilt illusion? Perception, 14, 275-283.

WENDEROTh, P.., \& White, D. (1979). Angle-matching and perceived line orientation. Perception, 8, 565-575.

WINER, B. J. (1962). Statistical principles in experimental design. New York: McGraw-Hill.

(Manuscript received November 11, 1985; revision accepted for publication March 21, 1986.) 